\title{
Stereoselective Synthesis of 2'-C-Methyl-cyclopropyl-fused
}

\section{Carbanucleosides as Potential Anti-HCV Agents}

Jeong A Lee, ${ }^{1}$ Hea Ok Kim, ${ }^{1}$ Dilip K. Tosh, ${ }^{1}$ Hyung Ryong Moon, ${ }^{2}$ Sanghee Kim, ${ }^{3}$ and Lak Shin Jeong ${ }^{1, *}$

${ }^{1}$ Laboratory of Medicinal Chemistry, College of Pharmacy, Ewha Womans University, Seoul 120-750, Korea, ${ }^{2}$ College of Pharmacy, Pusan National University, Busan 609735, Korea, and ${ }^{3}$ College of Pharmacy, Seoul National University, Seoul 151-742, Korea

\section{Supporting Information}

\section{Table of Contents}

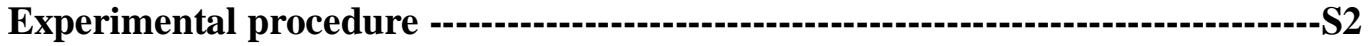

${ }^{1} \mathrm{H}$ Copies of 7, 10, 3a, and 3b ---:-'-S9

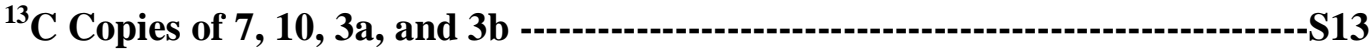




\section{General}

${ }^{1} \mathrm{H}$ NMR spectra $\left(\mathrm{CDCl}_{3}\right.$ or $\left.\mathrm{CD}_{3} \mathrm{OD}\right)$ were recorded on Varian Unity Inova $400 \mathrm{MHz}$. The ${ }^{1} \mathrm{H}$ NMR data are reported as peak multiplicities: s for singlet; $d$ for doublet; $d d$ for doublet of doublets; ddd for doublet of doublet of doublets; $t$ for triplet; pseudo $t$ for pseudo triplet; br s for broad singlet; and $\mathrm{m}$ for multiplet. Coupling constants are reported in hertz. ${ }^{13} \mathrm{C} \mathrm{NMR}$ spectra $\left(\mathrm{CDCl}_{3}\right.$ or $\left.\mathrm{CD}_{3} \mathrm{OD}\right)$ were recorded on Varian Unity Inova $100 \mathrm{MHz}$. The chemical shifts were reported as parts per million $(\delta)$ relative to the solvent peak. Optical rotations were determined on Jasco $\square$ in methylene chloride or methanol. UV spectra were recorded on U-3000 made by Histachi in methylene chloride or methanol. FAB mass spectra were recorded on Jeol HX 110 spectrometer. Elementary analyses were measured on EA1110. Melting points were measured on B540 made by Buchi. Reactions were checked with TLC (Merck precoated 60F254 plates). Spots were detected by viewing under a UV light, colorizing with charring after dipping in anisaldehyde solution with acetic acid and sulfuric acid and methanol. Column chromatography were performed on silica gel 60 (230-400 mesh, Merck). Reagents were purchased from Aldrich Chemical Company. Solvents were obtained from local suppliers. All the anhydrous solvents were distilled over $\mathrm{CaH}_{2}, \mathrm{P}_{2} \mathrm{O}_{5}$, or $\mathrm{Na} /$ benzophenone prior to the reaction.

(+)-(6aS, 3aS, 4S)-6-(tert-Butyldiphenylsilyloxymethyl)-2,2-dimethyl-4,6a-dihydro3aH-cyclopenta[1,3]dioxol-4-ol (5).

To the stirred solution of $4(9.09 \mathrm{~g}, 0.022 \mathrm{~mol})$ in methanol $(250 \mathrm{~mL})$, sodium borohydride $(0.92 \mathrm{mg}, 0.024 \mathrm{~mol})$ and cerium $(\square)$ chloride heptahydrate $(8.90 \mathrm{~g}, 0.024$ mol) was added at $0 \square$ and the reaction mixture was stirred at $0 \square$ for 30 minutes. The reaction mixture was partitioned between ethyl acetate and water and the organic layer was dried $\left(\mathrm{MgSO}_{4}\right)$, filtered, and evaporated. The residue was purified by silica gel column chromatography (hexane/ethyl acetate $=9 / 1)$ to give $5(7.63 \mathrm{~g}, 84 \%)$ as a colorless syrup: ${ }^{1} \mathrm{H}$ NMR $\left(\mathrm{CDCl}_{3}\right) \delta 1.08(\mathrm{~s}, 9 \mathrm{H}), 1.34(\mathrm{~s}, 3 \mathrm{H}), 1.36(\mathrm{~s}, 3 \mathrm{H}), 2.68$ (brs, $1 \mathrm{H}, \mathrm{OH}), 4.26-4.41(\mathrm{~m}, 2 \mathrm{H}), 4.56(\mathrm{~d}, 1 \mathrm{H}, J=2.4 \mathrm{~Hz}), 4.75(\mathrm{td}, 1 \mathrm{H}, J=0.4,5.6 \mathrm{~Hz})$, 4.87 (d, $1 \mathrm{H}, J=5.6 \mathrm{~Hz}$ ), 5.84 (pseudo t, $1 \mathrm{H}, J=0.8,1.2 \mathrm{~Hz}$ ), 7.35-7.45 (m, $6 \mathrm{H}$ ), 7.66$7.70(\mathrm{~m}, 4 \mathrm{H}) ;{ }^{13} \mathrm{C} \mathrm{NMR}\left(\mathrm{CDCl}_{3}\right) \delta 19.5,26.9,27.0,27.8,61.0,73.5,78.2,83.0,112.7$, 127.9, 129.5, 130.0, 133.5, 135.8, 145.5; MS (FAB) $m / z 447\left(\mathrm{M}+\mathrm{Na}^{+}\right) ;[\alpha]^{20}{ }_{\mathrm{D}}+28.6(c$ 0.66, $\mathrm{CH}_{2} \mathrm{Cl}_{2}$ ); Anal. Calcd for $\mathrm{C}_{25} \mathrm{H}_{32} \mathrm{O}_{4} \mathrm{Si}$ : C, 70.72; H, 7.60. Found: C, 70.39; H, 7.70. (+)-(1aR, 1bR, 4aS, 5S, 5aS)-1a-(tert-Butyldiphenylsilyloxymethyl)-3,3-dimethylhexahydro-2,4-dioxa-cyclopropa[a]pentalen-5-ol (6).

To a stirred solution of 5 (9.03 g, $21.27 \mathrm{mmol})$ in methylene chloride $(120 \mathrm{~mL})$ cooled 
in ice bath was added diethylzinc $(1.0 \mathrm{M}$ solution in hexane, $44.0 \mathrm{~mL})$ and the mixture was stirred at $0 \square$ for $30 \mathrm{~min}$. Diiodomethane ( $7 \mathrm{~mL}, 85.9 \mathrm{mmol}$ ) was added to the reaction mixture and the mixture was stirred at $0 \square$ for $1.5 \mathrm{~h}$ and stirred at room temperature for $5 \mathrm{~h}$. The mixture was quenched with cold aqueous ammonium chloride solution and extracted with methylene chloride. The organic layer was washed with aqueous saturated ammonium chloride, dried $\left(\mathrm{MgSO}_{4}\right)$, filtered, and evaporated. The residue was purified by silica gel column chromatography (hexane/ethyl acetate $=$ $100 / 1)$ to give $6(7.58 \mathrm{~g}, 81 \%)$ as a colorless syrup: ${ }^{1} \mathrm{H}$ NMR $\left(\mathrm{CDCl}_{3}\right) \delta 0.54(\mathrm{dd}, 1 \mathrm{H}, J$ = 5.6, $8.8 \mathrm{~Hz}), 1.05(\mathrm{~s}, 9 \mathrm{H}), 1.09(\mathrm{~m}, 1 \mathrm{H}), 1.31(\mathrm{~s}, 3 \mathrm{H}), 1.54(\mathrm{~s}, 3 \mathrm{H}), 1.61(\mathrm{~m}, 1 \mathrm{H})$, 3.29 (d, $1 \mathrm{H}, J=10.8 \mathrm{~Hz}), 4.07$ (d, $1 \mathrm{H}, J=11.2 \mathrm{~Hz}), 4.46(\mathrm{t}, 1 \mathrm{H}, J=6.0 \mathrm{~Hz}), 4.54$ (dt, $1 \mathrm{H}, J=0.8,6.8 \mathrm{~Hz}), 5.00(\mathrm{dd}, 1 \mathrm{H}, J=0.8,6.8 \mathrm{~Hz}), 7.35-7.45(\mathrm{~m}, 6 \mathrm{H}), 7.61-7.65$ (m, $4 \mathrm{H}) ;{ }^{13} \mathrm{C} \mathrm{NMR}\left(\mathrm{CDCl}_{3}\right) \delta 10.6,19.5,24.9,26.4,27.1,35.8,65.5,71.3,79.9,81.4$, 113.0, 127.9, 129.9, 130.0, 135.8, 135.8; MS (FAB) $m / z 461\left(\mathrm{M}+\mathrm{Na}^{+}\right) ;[\alpha]^{20}{ }_{\mathrm{D}}+39.2(c$ 0.73, $\mathrm{CH}_{2} \mathrm{Cl}_{2}$ ); Anal. Calcd for $\mathrm{C}_{26} \mathrm{H}_{34} \mathrm{O}_{4} \mathrm{Si}^{\mathrm{H}} \mathrm{H}_{2} \mathrm{O}: \mathrm{C}, 68.39 ; \mathrm{H}, 7.95$. Found: C, 68.67; H, 7.76 .

$(+)-(1 S, \quad 2 S, \quad 3 S, \quad 4 R, \quad 5 R)$-4-tert-Butoxy-5-(tert-butyldiphenylsilyloxymethyl)bicyclo[3.1.0]hexane-2,3-diol (7).

To a solution of $6(1.07 \mathrm{~g}, 2.44 \mathrm{mmol})$ in anhydrous methylene chloride $(100 \mathrm{~mL})$ at $78 \square$ was added trimethylaluminum $(2.0 \mathrm{M}$ solution in hexane, $3.4 \mathrm{~mL})$ and the reaction mixture was stirred at room temperature overnight. Another equivalent of trimethylaluminum (2.0 M solution in hexane, $6 \mathrm{~mL}$ ) was added and the mixture was stirred at room temperature for another $3 \mathrm{~d}$. After the reaction mixture was cooled to $78 \square$ and slowly quenched with an aqueous saturated ammonium chloride solution (caution!! highly exothermic), the resulting suspension was filtered and the filter cake was washed with methylene chloride several times. The combined filtrates were extracted with methylene chloride and the organic layer was washed with water, dried $\left(\mathrm{MgSO}_{4}\right)$, and evaporated. The residue was purified by silica gel column chromatography (hexane/ethyl acetate $=10 / 1)$ to give $7(0.66 \mathrm{~g}, 49 \%)$ as colorless syrup: ${ }^{1} \mathrm{H} \mathrm{NMR}\left(\mathrm{CDCl}_{3}\right) \delta 0.24(\mathrm{dd}, 1 \mathrm{H}, J=5.2,8.4 \mathrm{~Hz}), 1.08(\mathrm{~s}, 9 \mathrm{H}), 1.12(\mathrm{t}, 1 \mathrm{H}, J=$ $4.8 \mathrm{~Hz}$ ), 1.28 (s, $6 \mathrm{H}), 1.34$ (dd, $1 \mathrm{H}, J=4.4,8.4 \mathrm{~Hz}), 2.92$ (br s, $2 \mathrm{H}), 3.07$ (d, $1 \mathrm{H}, J=$ $11.2 \mathrm{~Hz}$ ), 3.84 (pseudo t, $1 \mathrm{H}, J=6.0,6.4 \mathrm{~Hz}$ ), 4.17 (d, $1 \mathrm{H}, J=10.8 \mathrm{~Hz}$ ), 4.32 (pseudo t, $1 \mathrm{H}, J=5.2,5.6 \mathrm{~Hz}), 4.58(\mathrm{~d}, 1 \mathrm{H}, J=6.4 \mathrm{~Hz}), 7.36-7.45(\mathrm{~m}, 6 \mathrm{H}), 7.63-7.68(\mathrm{~m}, 4 \mathrm{H})$; ${ }^{13} \mathrm{C}$ NMR $\left(\mathrm{CDCl}_{3}\right) \delta 8.8,19.3,27.0,27.8,28.3,35.2,64.7,69.8,71.0,72.2,74.5,127.7$, 129.8, 129.9, 133.5, 135.6; MS (FAB) $m / z 477\left(\mathrm{M}+\mathrm{Na}^{+}\right)$; $[\alpha]^{20}{ }_{\mathrm{D}}+37.6\left(c 0.25, \mathrm{CH}_{2} \mathrm{Cl}_{2}\right)$; Anal. Calcd for $\mathrm{C}_{27} \mathrm{H}_{38} \mathrm{O}_{4} \mathrm{Si} 1 / 3 \mathrm{H}_{2} \mathrm{O}$ : C, 70.39; H, 8.46. Found: C, 70.46; H, 8.48.

(+)-(1R, 2R, 3R, 4S, 5S)-2-tert-Butoxy-4-(tert-butyldiphenylsilyloxy)-1-(tert-butyl- 


\section{diphenylsilyloxymethyl)-bicyclo[3.1.0]hexan-3-ol (8).}

To the solution of 7 (2.83 g, $6.23 \mathrm{mmol})$ in methylene chloride $(25 \mathrm{~mL})$ were added imidazole (1.27 g, $18.5 \mathrm{mmol})$ and tert-butyldiphenylsilyl chloride $(2.15 \mathrm{~mL}, 8.23$ mmol) and the reaction mixture was stirred at room temperature for $20 \mathrm{~min}$ and evaporated. The residue was dissolved in ethyl acetate and the organic layer was washed with brine, dried $\left(\mathrm{MgSO}_{4}\right)$, filtered, and evaporated. The residue was purified by silica gel column chromatography (hexane/ethyl acetate $=30 / 1)$ to give $8(3.89 \mathrm{~g}, 90 \%)$ as colorless syrup: ${ }^{1} \mathrm{H}$ NMR $\left(\mathrm{CDCl}_{3}\right) \delta 0.28(\mathrm{dd}, 1 \mathrm{H}, J=4.8,8.4 \mathrm{~Hz}), 0.95-1.00(\mathrm{~m}, 10 \mathrm{H})$, 1.10 (s, $9 \mathrm{H}$ ), 1.23 (s, $9 \mathrm{H}$ ), 1.52 (pseudo t, $1 \mathrm{H}, J=4.4,4.8 \mathrm{~Hz}$ ), 2.97 (d, $1 \mathrm{H}, J=11.2$ $\mathrm{Hz}), 3.75$ (t, $1 \mathrm{H}, J=6.0 \mathrm{~Hz}), 4.08(\mathrm{~d}, 1 \mathrm{H}, J=10.8 \mathrm{~Hz}), 4.31(\mathrm{~d}, 1 \mathrm{H}, J=6.4 \mathrm{~Hz}), 4.39$ (pseudo t, $1 \mathrm{H}, J=5.2,5.6 \mathrm{~Hz}), 7.24-7.60(\mathrm{~m}, 16 \mathrm{H}), 7.73-7.79(\mathrm{~m}, 4 \mathrm{H}) ;{ }^{13} \mathrm{C} \mathrm{NMR}$ $\left(\mathrm{CDCl}_{3}\right) \delta 10.2,19.4,19.7,27.0,27.1,27.2,28.6,35.6,65.6,71.7,73.1,73.7,73.8,76.9$, 77.2, 77.5, 127.7, 127.8, 127.8, 127.8, 129.8, 133.8, 133.8, 134.2, 134.4, 135.7, 135.7, 136.0, 136.1; MS (FAB) $\mathrm{m} / \mathrm{z} 693(\mathrm{M}) ;[\alpha]^{20}{ }_{\mathrm{D}}+27.3\left(c 0.11, \mathrm{CH}_{2} \mathrm{Cl}_{2}\right.$ ); Anal. Calcd for $\mathrm{C}_{43} \mathrm{H}_{56} \mathrm{O}_{4} \mathrm{Si}_{2}$ : C, 74.52; H, 8.14. Found: C, 74.33; H, 8.25.

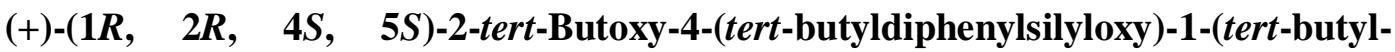
diphenylsilyloxymethyl)-bicyclo[3.1.0]hexan-3-one (9).

Dimethyl sulfoxide $(1.60 \mathrm{~mL}, 22.5 \mathrm{mmol})$ was added slowly to the solution of oxalyl chloride $(2.0 \mathrm{M}$ solution in methylene chloride, $5.0 \mathrm{~mL})$ in methylene chloride $(30 \mathrm{~mL})$ at $-78 \square$ and the reaction mixture was stirred at $-78 \square$ for 40 minutes. To this reaction mixture was added a solution of alcohol 8 (3.87 g, $5.58 \mathrm{mmol})$ in methylene chloride (30 $\mathrm{mL}$ ) at $-78 \square$ and the reaction mixture was stirred for $1 \mathrm{~h}$ at the same temperature. After triethylamine $(6.0 \mathrm{~mL}, 42.8 \mathrm{mmol})$ was added to the mixture at $-78 \square$, the reaction mixture was further stirred at room temperature overnight. After adding saturated aqueous $\mathrm{NH}_{4} \mathrm{Cl}$ solution carefully at $0 \square$, the reaction mixture was partitioned between methylene chloride and water. The organic layer was dried $\left(\mathrm{MgSO}_{4}\right)$, filtered, and evaporated. The residue was purified by silica gel column chromatography (hexane/ethyl acetate $=40 / 1)$ to give $9(3.10 \mathrm{~g}, 80 \%)$ as colorless syrup: ${ }^{1} \mathrm{H}$ NMR $\left(\mathrm{CDCl}_{3}\right) \delta 0.53(\mathrm{~m}, 1 \mathrm{H}), 0.83(\mathrm{dd}, 1 \mathrm{H}, J=4.0,6.0 \mathrm{~Hz}), 1.03(\mathrm{~s}, 9 \mathrm{H}), 1.12(\mathrm{~s}, 9 \mathrm{H})$, $1.25(\mathrm{~m}, 1 \mathrm{H}), 1.29(\mathrm{~s}, 9 \mathrm{H}), 3.04(\mathrm{~d}, 1 \mathrm{H}, J=11.2 \mathrm{~Hz}), 4.23(\mathrm{~d}, 1 \mathrm{H}, J=11.2 \mathrm{~Hz}), 4.63$ $(\mathrm{td}, 1 \mathrm{H}, J=1.6,5.6 \mathrm{~Hz}), 4.76(\mathrm{t}, 1 \mathrm{H}, J=2.0 \mathrm{~Hz}), 7.21-7.62(\mathrm{~m}, 14 \mathrm{H}), 7.75-7.85(\mathrm{~m}, 4$ $\mathrm{H}) ;{ }^{13} \mathrm{C} \mathrm{NMR}\left(\mathrm{CDCl}_{3}\right) \delta 9.4,19.4,19.5,23.5,26.9,27.1,28.5,30.6,60.5,73.3,74.6$, 75.0, 127.8, 127.8, 127.9, 129.9, 129.9, 130.0, 133.4, 133.4, 134.2, 135.6, 135.7, 136.0, 136.1; MS (FAB) $m / z 692\left(\mathrm{M}+\mathrm{H}^{+}\right)$; $[\alpha]^{20}{ }_{\mathrm{D}}+14.2\left(c 0.26, \mathrm{CH}_{2} \mathrm{Cl}_{2}\right)$; Anal. Calcd for $\mathrm{C}_{43} \mathrm{H}_{54} \mathrm{O}_{4} \mathrm{Si}_{2}:$ C, 74.73; H, 7.88. Found: C, 74.59; H, 7.59.

(+)-(1R, 2R, 3R, 4S, 5S)-2-tert-Butoxy-4-(tert-butyldiphenylsilyloxy)-1-(tert-butyl- 
diphenylsilyloxymethyl)-3-methyl-bicyclo[3.1.0]hexan-3-ol (10).

To a stirring solution of $9(3.10 \mathrm{~g}, 4.48 \mathrm{mmol})$ in ether $(15 \mathrm{~mL})$ was added methyl magnesium iodide $(5.8 \mathrm{~mL}, 17.4 \mathrm{mmol})$ at $0 \square$ and the mixture was stirred at $0 \square$ for $1.5 \mathrm{~h}$ and then at room temperature for $2.5 \mathrm{~h}$. Water was added to the reaction mixture and the reaction mixture was extracted with methylene chloride. The organic layer was washed with brine, dried $\left(\mathrm{MgSO}_{4}\right)$, filtered, and evaporated. The residue was purified by silica gel column chromatography (hexane/ethyl acetate $=40 / 1)$ to give $\mathbf{1 0}(2.86 \mathrm{~g}$, 90\%) as yellowish syrup: ${ }^{1} \mathrm{H} \mathrm{NMR}\left(\mathrm{CDCl}_{3}\right) \delta 0.35(\mathrm{dd}, 1 \mathrm{H}, J=4.8,8.8 \mathrm{~Hz}), 1.02(\mathrm{~s}, 9$ H), $1.10(\mathrm{~s}, 9 \mathrm{H}), 1.20(\mathrm{~s}, 9 \mathrm{H}), 1.22-1.27(\mathrm{~m}, 1 \mathrm{H}), 1.62(\mathrm{t}, 1 \mathrm{H}, J=4.4 \mathrm{~Hz}), 3.15(\mathrm{~d}, 1$ $\mathrm{H}, J=11.2 \mathrm{~Hz}$ ), 3.54 (br s, $1 \mathrm{H}, \mathrm{OH}), 3.82(\mathrm{~d}, 1 \mathrm{H}, J=0.8 \mathrm{~Hz}$ ), 3.96 (dd, $1 \mathrm{H}, J=1.2$, $5.6 \mathrm{~Hz}), 3.98$ (d, $1 \mathrm{H}, J=10.8 \mathrm{~Hz}), 7.32-7.65(\mathrm{~m}, 16 \mathrm{H}), 7.76-7.83(\mathrm{~m}, 4 \mathrm{H}) ;{ }^{13} \mathrm{C} \mathrm{NMR}$ $\left(\mathrm{CDCl}_{3}\right) \delta 11.2,19.4,19.8,24.8,27.1,27.2,28.9,29.2,36.3,66.7,73.6,76.9,78.6,80.5$, 127.6, 127.6, 127.8, 127.9, 129.6, 129.6, 129.8, 129.9, 133.6, 133.6, 134.5, 134.7, 135.8, 135.9, 136.2, 136.3; MS (FAB) $m / z 730\left(\mathrm{M}+\mathrm{Na}^{+}\right) ;[\alpha]^{20}{ }_{\mathrm{D}}+21.0\left(c 0.61, \mathrm{CH}_{2} \mathrm{Cl}_{2}\right)$; Anal. Calcd for $\mathrm{C}_{44} \mathrm{H}_{58} \mathrm{O}_{4} \mathrm{Si}_{2} \cdot 1 / 3 \mathrm{H}_{2} \mathrm{O}: \mathrm{C}, 74.11 ; \mathrm{H}, 8.29$. Found: C, 74.30; H, 8.30.

$(-)-(1 S, \quad 2 S, \quad 3 S, \quad 4 R, \quad 5 R)$-4-tert-Butoxy-5-hydroxymethyl-3-methylbicyclo[3.1.0]hexane-2,3-diol (11).

To the stirred solution of $\mathbf{1 0}(2.83 \mathrm{~g}, 4.00 \mathrm{mmol})$ in THF $(20 \mathrm{~mL})$ was added tetrabutylammonium fluoride $(1.0 \mathrm{M}$ solution in THF, $28 \mathrm{~mL})$ and the reaction mixture was stirred at room temperature overnight. After evaporation, the residue was purified by silica gel column chromatography (methylene chloride/methanol $=50 / 1$ ) to give $\mathbf{1 1}$ $(0.89 \mathrm{~g}, 97 \%)$ as yellowish syrup: ${ }^{1} \mathrm{H} \mathrm{NMR}\left(\mathrm{CD}_{3} \mathrm{OD}\right) \delta 0.33(\mathrm{~m}, 1 \mathrm{H}), 1.20(\mathrm{~s}, 3 \mathrm{H})$, 1.28 (s, $9 \mathrm{H}), 1.39$ (t, $1 \mathrm{H}, J=4.4 \mathrm{~Hz}), 1.49$ (m, $1 \mathrm{H}), 3.00$ (d, $1 \mathrm{H}, J=12.0 \mathrm{~Hz}), 3.92$ (d, $1 \mathrm{H}, J=4.8 \mathrm{~Hz}), 4.01(\mathrm{~d}, 1 \mathrm{H}, J=11.6 \mathrm{~Hz}), 4.14(\mathrm{~s}, 1 \mathrm{H}) ;{ }^{13} \mathrm{C} \mathrm{NMR}\left(\mathrm{CD}_{3} \mathrm{OD}\right) \delta 9.9$, 27.1, 28.6, 29.7, 35.6, 65.2, 75.2, 76.5, 77.0, 77.9; $\mathrm{MS}(\mathrm{FAB}) \mathrm{m} / \mathrm{z} 253\left(\mathrm{M}+\mathrm{Na}^{+}\right)$; $[\alpha]^{20}{ }_{\mathrm{D}}-$ 10.9 ( $c 0.11, \mathrm{CH}_{2} \mathrm{Cl}_{2}$ ); Anal. Calcd for $\mathrm{C}_{12} \mathrm{H}_{22} \mathrm{O}_{4}: \mathrm{C}, 62.58 ; \mathrm{H}, 9.63$. Found: $\mathrm{C}, 62.18 ; \mathrm{H}$, 9.50 .

(+)-(1S, 2S, 3S, 4R, 5R)-4-tert-Butoxy-5-(tert-butyl-dimethyl-silanyloxymethyl)-3methyl-bicyclo[3.1.0]hexane-2,3-diol (12).

To the stirred solution of $11(0.29 \mathrm{~g}, 1.24 \mathrm{mmol})$ in methylene chloride were added imidazole $(0.21 \mathrm{~g}, 3.12 \mathrm{mmol})$ and tert-butyldimethylsilyl chloride $(0.22 \mathrm{~g}, 1.40 \mathrm{mmol})$ at $0 \square$ and the reaction mixture was stirred at $0 \square$ for $2 \mathrm{~h}$. The reaction mixture was partitioned between methylene chloride and water. The organic layer was washed with brine, dried $\left(\mathrm{MgSO}_{4}\right)$, filtered, and evaporated. The residue was purified by silica gel column chromatography (hexane/ethyl acetate $=5 / 1)$ to give $12(0.28 \mathrm{~g}, 65 \%)$ as yellowish syrup: ${ }^{1} \mathrm{H}$ NMR $\left(\mathrm{CDCl}_{3}\right) \delta 0.02(\mathrm{~s}, 6 \mathrm{H}), 0.33(\mathrm{dd}, 1 \mathrm{H}, J=5.2,8.4 \mathrm{~Hz}), 0.88$ 
(s, $9 \mathrm{H}), 1.17(\mathrm{t}, 1 \mathrm{H}, J=4.8 \mathrm{~Hz}), 1.20(\mathrm{~s}, 3 \mathrm{H}), 1.25(\mathrm{~s}, 9 \mathrm{H}), 1.48(\mathrm{~m}, 1 \mathrm{H}), 2.97(\mathrm{~d}, 1 \mathrm{H}$, $J=10.8 \mathrm{~Hz}), 3.90(\mathrm{~d}, 1 \mathrm{H}, J=5.6 \mathrm{~Hz}), 4.03(\mathrm{~d}, 1 \mathrm{H}, J=10.8 \mathrm{~Hz}), 4.11(\mathrm{~s}, 1 \mathrm{H}) ;{ }^{13} \mathrm{C}$ $\operatorname{NMR}\left(\mathrm{CDCl}_{3}\right) \delta-5.2,-5.0,8.4,18.4,26.0,26.9,29.2,29.3,35.2,64.8,74.4,75.5,75.8$, 76.9; MS (FAB) $m / z 367\left(\mathrm{M}+\mathrm{Na}^{+}\right) ;[\alpha]^{20}{ }_{\mathrm{D}}+52.0\left(c 0.05, \mathrm{CH}_{2} \mathrm{Cl}_{2}\right)$; Anal. Calcd for $\mathrm{C}_{18} \mathrm{H}_{36} \mathrm{O}_{4} \mathrm{Si}: \mathrm{C}, 62.74 ; \mathrm{H}, 10.53$. Found: C, 62.49; H, 10.57 .

(+)-(1aS, 3S, 4R, 5R, 5aR)-(5-tert-Butoxy-4a-methyl-3-oxo-tetrahydro-2,4-dioxa$3 \lambda^{4}$-thia-cyclopropa[a]pentalen-5a-ylmethoxy)-tert-butyldimethylsilane (13).

To a solution of $12(0.69 \mathrm{~g}, 1.99 \mathrm{mmol})$ in methylene chloride $(5 \mathrm{~mL})$ were added triethylamine $(1.0 \mathrm{~mL}, 7.14 \mathrm{mmol})$ and thionyl chloride $(0.22 \mathrm{~mL}, 3.02 \mathrm{mmol})$ at $0 \square$ and the reaction mixture was stirred at $0 \square$ for $10 \mathrm{~min}$ and partitioned between methylene chloride and water. The organic layer was washed with brine, dried $\left(\mathrm{MgSO}_{4}\right)$, filtered, and evaporated. The residue was purified by silica gel column chromatography (hexane/ethyl acetate $=10 / 1)$ to give $13(0.75 \mathrm{~g}, 96 \%)$ as a diastereomeric mixture: ${ }^{1} \mathrm{H}$ NMR $\left(\mathrm{CDCl}_{3}\right)$ (for major isomer) $\delta 0.05(\mathrm{~m}, 6 \mathrm{H}), 0.62(\mathrm{dd}, 1 \mathrm{H}, J=5.6,8.8 \mathrm{~Hz}), 0.90$ (s, $9 \mathrm{H}), 1.18(\mathrm{~s}, 9 \mathrm{H}), 1.42(\mathrm{dd}, 1 \mathrm{H}, J=4.4,5.6 \mathrm{~Hz}), 1.58(\mathrm{~m}, 1 \mathrm{H}), 1.67(\mathrm{~s}, 3 \mathrm{H}), 3.00$ $(\mathrm{d}, 1 \mathrm{H}, J=10.8 \mathrm{~Hz}), 3.95$ (s, $1 \mathrm{H}), 4.04$ (d, $1 \mathrm{H}, J=10.8 \mathrm{~Hz}), 5.01$ (dd, $1 \mathrm{H}, J=1.2$, $5.6 \mathrm{~Hz}$ ); ${ }^{13} \mathrm{C} \mathrm{NMR}\left(\mathrm{CDCl}_{3}\right.$ ) (for major isomer) $\delta-5.3,-5.2,10.9,18.5,23.0,23.8,26.0$ $26.1,28.8,29.1,40.5,65.3,74.3,75.4,90.1,97.3$; MS (FAB) $m / z 391\left(\mathrm{M}+\mathrm{H}^{+}\right) ;[\alpha]^{20}{ }_{\mathrm{D}}$ $+16.4\left(c 0.11, \mathrm{CH}_{2} \mathrm{Cl}_{2}\right)$.

(-)-(1R, 2R, 3S, 4R, 5S)-4-(6-Amino-purin-9-yl)-2-tert-butoxy-1-hydroxymethyl-3methyl-bicyclo[3.1.0]hexan-3-ol (15).

To a solution of $\mathbf{1 3}(0.34 \mathrm{~g}, 0.87 \mathrm{mmol})$ in carbon tetrachloride, acetonitrile, and water $(1: 1: 1.5,5.6 \mathrm{~mL})$ were added sodium metaperiodate $(0.40 \mathrm{~g}, 1.26 \mathrm{mmol})$ and ruthenium chloride trihydrate $(8.9 \mathrm{mg})$ and the reaction mixture was stirred at room temperature for $10 \mathrm{~min}$. The reaction mixture was partitioned between ether and water and the organic layer was washed with brine and saturated aqueous sodium bicarbonate solution, dried $\left(\mathrm{MgSO}_{4}\right)$, filtered, and evaporated. The residue was filtered through a short silica gel pad using hexane and ethyl acetate $(1: 2)$ as the eluent to give crude $14(0.40 \mathrm{~g})$ as yellowish syrup, which was immediately used for the next reaction.

A suspension of adenine and sodium hydride in DMF was stirred to $80 \square$ for $3 \mathrm{~h}$. To this solution was added a solution of crude $14(0.40 \mathrm{~g})$ in DMF $(3 \mathrm{~mL})$ and the mixture was heated to $70 \square$ for $4 \mathrm{~h}$. The mixture was cooled down to room temperature and $20 \%$ sulfuric acid was added slowly. The reaction mixture was stirred at $70 \square$ for $2 \mathrm{~h}$, then neutralized with $1 \mathrm{~N} \mathrm{NaOH}$ solution, and evaporated. The residue was purified by silica gel column chromatography (methylene chloride/ methanol $=30 / 1$ ) to give $\mathbf{1 5}$ $(0.18 \mathrm{~g}, 58 \%)$ as a white solid after recrystallization from ether and methanol: $\mathrm{mp} 277$ - 
$283 \square$; UV $(\mathrm{MeOH}) \lambda_{\max } 261.0 \mathrm{~nm} ;{ }^{1} \mathrm{H} \mathrm{NMR}\left(\mathrm{CD}_{3} \mathrm{OD}\right) \delta 0.68$ (ddd, $1 \mathrm{H}, J=1.6,4.0$, $7.6 \mathrm{~Hz}), 0.75$ (s, $3 \mathrm{H}), 1.32$ (s, $9 \mathrm{H}), 1.59$ (m, $2 \mathrm{H}), 3.17$ (d, $1 \mathrm{H}, J=11.6 \mathrm{~Hz}), 4.26$ (d, 1 $\mathrm{H}, J=11.2 \mathrm{~Hz}), 4.53(\mathrm{~d}, 1 \mathrm{H}, J=1.2 \mathrm{~Hz}), 4.79(\mathrm{~s}, 1 \mathrm{H}), 8.19(\mathrm{~s}, 1 \mathrm{H}), 8.53(\mathrm{~s}, 1 \mathrm{H}) ;{ }^{13} \mathrm{C}$ NMR $\left(\mathrm{CD}_{3} \mathrm{OD}\right) \delta 13.0,24.3,25.9,29.8,37.3,65.3,66.2,75.7,76.6,82.1,120.4,142.7$, 150.8, 153.7, 157.7; MS (FAB) $m / z 348\left(\mathrm{M}+\mathrm{H}^{+}\right)$; $[\alpha]^{20}{ }_{\mathrm{D}}-100.0\left(c 0.03, \mathrm{CH}_{3} \mathrm{OH}\right)$; Anal. Calcd for $\mathrm{C}_{17} \mathrm{H}_{25} \mathrm{~N}_{5} \mathrm{O}_{3} 0.2 \mathrm{C}_{2} \mathrm{H}_{5} \mathrm{OC}_{2} \mathrm{H}_{5}: \mathrm{C}, 59.02 ; \mathrm{H}, 7.51 ; \mathrm{N}, 19.33$. Found: $\mathrm{C}, 58.92 ; \mathrm{H}$, 7.36; N, 19.37 .

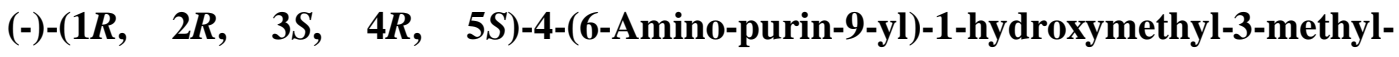
bicyclo[3.1.0]hexane-2,3-diol (3a).

A mixture of $15(0.15 \mathrm{~g}, 0.42 \mathrm{mmol})$ and $70 \%$ aqueous trifluoroacetic acid $(1.5 \mathrm{~mL})$ was stirred at room temperature for $50 \mathrm{~min}$ and evaporated. The residue was coevaporated with methanol and toluene. The residue was purified by silica gel column chromatography (methylene chloride/methanol $=7 / 1$ ) to give $\mathbf{3 a}(0.11 \mathrm{~g}, 92 \%$ ) as a white solid after recrystallization from methanol: mp 244-247 $\square$; UV (MeOH) $\lambda_{\max }$ $261.0 \mathrm{~nm} ;{ }^{1} \mathrm{H}$ NMR $\left(\mathrm{CD}_{3} \mathrm{OD}\right) \delta 0.69$ (m, $\left.1 \mathrm{H}\right), 0.70$ (s, $\left.3 \mathrm{H}\right), 1.59$ (dd, $1 \mathrm{H}, J=4.0,8.8$ $\mathrm{Hz}), 1.76(\mathrm{dd}, 1 \mathrm{H}, J=4.0,4.8 \mathrm{~Hz}), 3.26(\mathrm{~d}, 1 \mathrm{H}, J=11.6 \mathrm{~Hz}), 4.31(\mathrm{~d}, 1 \mathrm{H}, J=11.6$ $\mathrm{Hz}), 4.46(\mathrm{~d}, 1 \mathrm{H}, J=1.2 \mathrm{~Hz}), 4.86(\mathrm{~s}, 1 \mathrm{H}), 8.20(\mathrm{~s}, 1 \mathrm{H}), 8.56(\mathrm{~s}, 1 \mathrm{H}) ;{ }^{13} \mathrm{C} \mathrm{NMR}$ $\left(\mathrm{CD}_{3} \mathrm{OD}\right) \delta 12.6,23.8,24.6,37.7,65.1,65.5,76.9,80.8,120.2,142.1,150.9,153.8$, 157.7; MS (FAB) $m / z 292\left(\mathrm{M}+\mathrm{H}^{+}\right) ;[\alpha]^{20}{ }_{\mathrm{D}}-55.0\left(c\right.$ 0.02, $\left.\mathrm{CH}_{3} \mathrm{OH}\right)$; Anal. Calcd for $\mathrm{C}_{13} \mathrm{H}_{17} \mathrm{~N}_{5} \mathrm{O}_{3}$ : C, 53.60; H, 5.88; N, 24.04. Found: C, 53.92; H, 5.85; N, 23.84.

$(-)-(1 R, \quad 2 R, \quad 3 S, \quad 4 R, \quad 5 S)-4-(2-A m i n o-6-c h l o r o-p u r i n-9-y l)-2-t e r t-b u t o x y-1-$ hydroxymethyl-3-methyl-bicyclo[3.1.0]hexan-3-ol (16).

Cyclic sulfite $13(0.30 \mathrm{~g}, 0.76 \mathrm{mmol})$ was converted to $16(0.17 \mathrm{~g}, 58 \%)$ as a white solid and its $N^{7}$-isomer $(0.040 \mathrm{~g}, 14 \%)$ as a white solid according to the same procedure used in the preparation of $\mathbf{1 5 .}$

$N^{9}$-isomer (16): mp 115-121 $\square$; UV (MeOH) $\lambda_{\max } 310.5 \mathrm{~nm} ;{ }^{1} \mathrm{H}$ NMR $\left(\mathrm{CD}_{3} \mathrm{OD}\right) \delta 0.67$ (ddd, $1 \mathrm{H}, J=1.6,5.2,8.8 \mathrm{~Hz}), 0.80$ (s, $3 \mathrm{H}), 1.31$ (s, $9 \mathrm{H}), 1.53$ (dd, $1 \mathrm{H}, J=4.0,8.8$ $\mathrm{Hz}$ ), 1.61 (pseudo t, $1 \mathrm{H}, J=4.4,5.2 \mathrm{~Hz}), 3.14$ (d, $1 \mathrm{H}, J=11.2 \mathrm{~Hz}), 4.27$ (d, $1 \mathrm{H}, J=$ $11.6 \mathrm{~Hz}), 4.45(\mathrm{~d}, 1 \mathrm{H}, J=1.2 \mathrm{~Hz}), 4.74(\mathrm{~s}, 1 \mathrm{H}), 8.67(\mathrm{~s}, 1 \mathrm{H}) ;{ }^{13} \mathrm{C} \mathrm{NMR}\left(\mathrm{CD}_{3} \mathrm{OD}\right) \delta$ 11.6, 23.1, 24.3, 28.4, 35.8, 63.3, 63.6, 74.3, 75.1, 80.7, 123.5, 142.4, 150.6, 154.3, 160.4; MS (FAB) $m / z 382\left(\mathrm{M}+\mathrm{H}^{+}\right) ;[\alpha]^{20}{ }_{\mathrm{D}}-12.5\left(c\right.$ 0.04, $\left.\mathrm{CH}_{3} \mathrm{OH}\right)$; Anal. Calcd for $\mathrm{C}_{17} \mathrm{H}_{24} \mathrm{ClN}_{5} \mathrm{O}_{3} \cdot 0.2 \mathrm{C}_{2} \mathrm{H}_{5} \mathrm{OC}_{2} \mathrm{H}_{5}: \mathrm{C}, 53.89 ; \mathrm{H}, 6.61 ; \mathrm{N}, 17.65$. Found: C, 53.54; H, 6.46; $\mathrm{N}, 17.25$.

$N^{7}$-isomer: mp 120-130 $\square$; UV (MeOH) $\lambda_{\max } 286.0 \mathrm{~nm} ;{ }^{1} \mathrm{H} \mathrm{NMR}\left(\mathrm{CD}_{3} \mathrm{OD}\right) \delta 0.63$ (ddd, $1 \mathrm{H}, J=1.2,4.8,8.8 \mathrm{~Hz}), 0.77(\mathrm{~s}, 3 \mathrm{H}), 1.31(\mathrm{~s}, 9 \mathrm{H}), 1.47$ (dd, $1 \mathrm{H}, J=4.0,8.8 \mathrm{~Hz})$, 1.56 (pseudo t, $1 \mathrm{H}, J=4.4,4.8 \mathrm{~Hz}), 3.11$ (d, $1 \mathrm{H}, J=11.6 \mathrm{~Hz}), 4.25$ (d, $1 \mathrm{H}, J=12.0$ 
$\mathrm{Hz}), 4.52(\mathrm{~d}, 1 \mathrm{H}, J=1.2 \mathrm{~Hz}), 4.63(\mathrm{~s}, 1 \mathrm{H}), 8.15(\mathrm{~s}, 1 \mathrm{H}) ;{ }^{13} \mathrm{C} \mathrm{NMR}\left(\mathrm{CD}_{3} \mathrm{OD}\right) \delta$ 11.6, 23.3, 24.5, 28.5, 35.9, 63.7, 64.0, 74.2, 75.4, 80.7, 114.3, 136.8, 152.4, 155.5, 159.9; MS (FAB) $m / z 404\left(\mathrm{M}+\mathrm{Na}^{+}\right) ;[\alpha]^{20}{ }_{\mathrm{D}}-9.0\left(c \mathrm{c} 0.93, \mathrm{CH}_{3} \mathrm{OH}\right)$.

(+)-(1R, 2R, 3S, 4R, 5S)-2-Amino-9-(3,4-dihydroxy-5-hydroxymethyl-3-methylbicyclo[3.1.0]hex-2-yl)-1,9-dihydro-purin-6-one (3b)

Compound 16 (99.5 $\mathrm{mg}, 0.26 \mathrm{mmol}$ ) was converted to 2-amino-6-chloropurine derivative $(85 \mathrm{mg}, 100 \%)$ as a white solid according to the same procedure used in the preparation of 3a: mp 192-197 $\square$; UV (MeOH) $\lambda_{\max } 310.5 \mathrm{~nm} ;{ }^{1} \mathrm{H}$ NMR $\left(\mathrm{CD}_{3} \mathrm{OD}\right) \delta$ $0.68(\mathrm{ddd}, 1 \mathrm{H}, J=1.2,5.2,8.5 \mathrm{~Hz}), 0.74(\mathrm{~s}, 3 \mathrm{H}), 1.53(\mathrm{dd}, 1 \mathrm{H}, J=4.0,8.8 \mathrm{~Hz}), 1.75$ $(\mathrm{dd}, 1 \mathrm{H}, J=4.0,5.2 \mathrm{~Hz}), 3.25$ (d, $1 \mathrm{H}, J=11.6 \mathrm{~Hz}), 4.29$ (d, $1 \mathrm{H}, J=11.6 \mathrm{~Hz}), 4.41$ (d, $1 \mathrm{H}, J=1.6 \mathrm{~Hz}), 4.80(\mathrm{~s}, 1 \mathrm{H}), 8.62(\mathrm{~s}, 1 \mathrm{H}) ;{ }^{13} \mathrm{C} \mathrm{NMR}\left(\mathrm{CD}_{3} \mathrm{OD}\right) \delta 12.5,23.8,24.4$, 37.6, 64.4, 64.8, 76.9, 80.7, 124.9, 143.4, 151.9, 155.6, 161.8; MS (FAB) $\mathrm{m} / z 326$ $\left(\mathrm{M}+\mathrm{H}^{+}\right) ;[\alpha]^{20}{ }_{\mathrm{D}}+22.9\left(c 0.41, \mathrm{CH}_{3} \mathrm{OH}\right)$; Anal. Calcd for $\mathrm{C}_{13} \mathrm{H}_{16} \mathrm{ClN}_{5} \mathrm{O}_{3} \cdot 1 / 3 \mathrm{CH}_{2} \mathrm{Cl}_{2}: \mathrm{C}$, 45.23; H, 4.74; N, 19.78. Found: C, 45.53; H, 4.85; N, 19.83.

A solution of 2-amino-6-chloropurine derivative $(59.9 \mathrm{mg}, 0.18 \mathrm{mmol}$ ) and $3 \mathrm{~N}$ aqueous $\mathrm{HCl}(10 \mathrm{~mL})$ was stirred at room temperature for $2 \mathrm{~d}$. After dried under the reduced pressure, the residue was coevaporated with methanol and toluene. The residue was purified by reverse column chromatography using methanol and water as the eluent to give $3 \mathbf{b}$ (48.1 mg, 85\%) as a white solid. mp 216-233 $\square$; UV (MeOH) $\lambda_{\max } 255.0 \mathrm{~nm}$; ${ }^{1} \mathrm{H}$ NMR $\left(\mathrm{CD}_{3} \mathrm{OD}\right) \delta 0.65(\mathrm{ddd}, 1 \mathrm{H}, J=1.6,5.2,8.8 \mathrm{~Hz}), 0.76(\mathrm{~s}, 3 \mathrm{H}), 1.49(\mathrm{dd}, 1 \mathrm{H}, J$ $=4.0,8.8 \mathrm{~Hz}), 1.71(\mathrm{dd}, 1 \mathrm{H}, J=4.0,4.8 \mathrm{~Hz}), 3.23(\mathrm{~d}, 1 \mathrm{H}, J=11.6 \mathrm{~Hz}), 4.27(\mathrm{~d}, 1 \mathrm{H}, J$ $=11.6 \mathrm{~Hz}), 4.39(\mathrm{~d}, 1 \mathrm{H}, J=1.2 \mathrm{~Hz}), 4.70(\mathrm{~s}, 1 \mathrm{H}), 8.25(\mathrm{~s}, 1 \mathrm{H}) ;{ }^{13} \mathrm{C} \mathrm{NMR}\left(\mathrm{CD}_{3} \mathrm{OD}\right) \delta$ 12.4, 23.9, 24.5, 37.5, 64.1, 64.9, 77.0, 80.9, 117.7, 138.5, 153.5, 155.7, 160.0; MS (FAB) $m / z 330\left(\mathrm{M}+\mathrm{Na}^{+}\right) ;[\alpha]^{20}{ }_{\mathrm{D}}+62.3\left(c 0.43, \mathrm{CH}_{3} \mathrm{OH}\right)$; Anal. Calcd for $\mathrm{C}_{13} \mathrm{H}_{17} \mathrm{~N}_{5} \mathrm{O}_{4}: \mathrm{C}$, 50.81; H, 5.58; N, 22.79. Found: C, 51.37; H, 5.71; N, 22.71. 


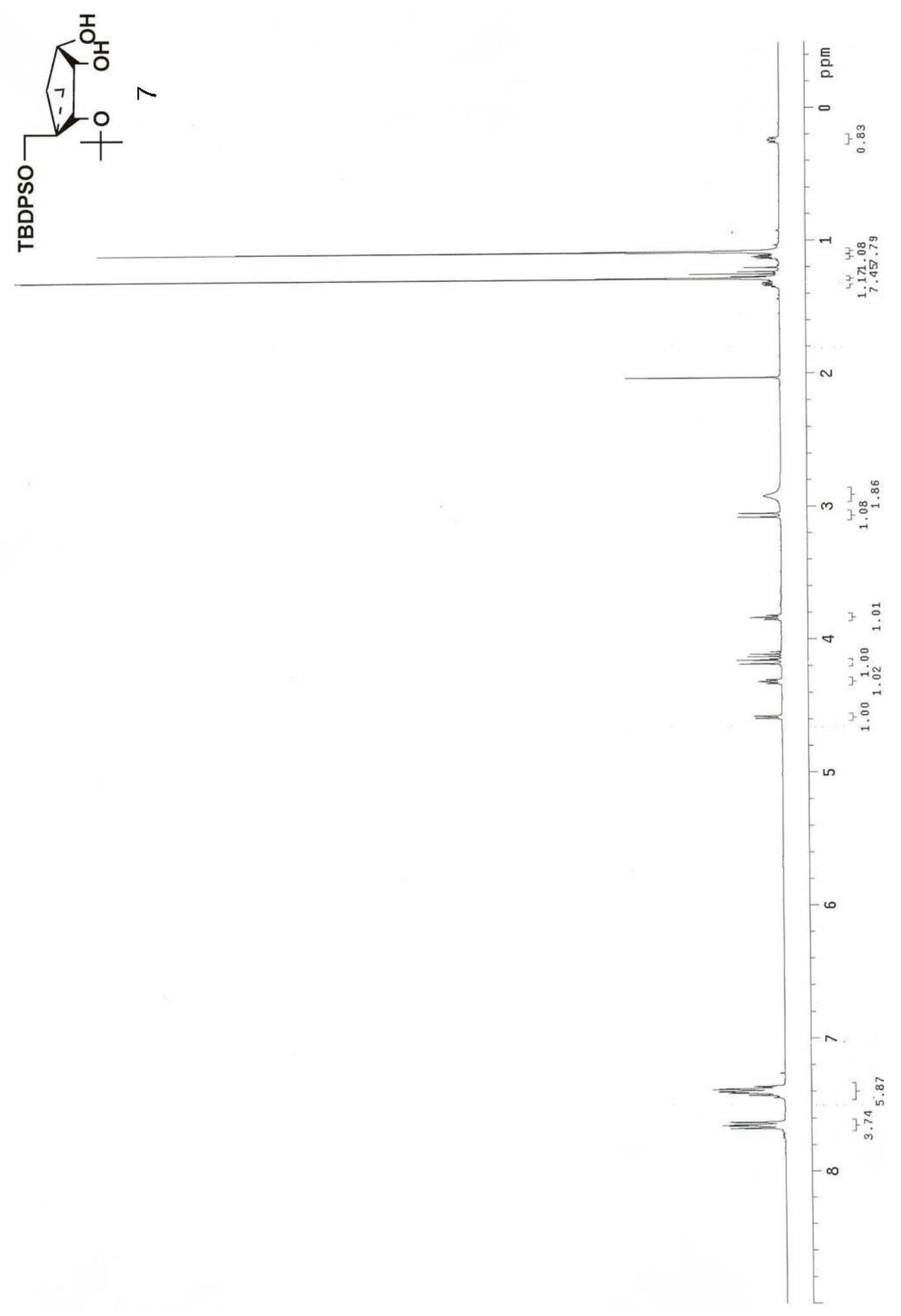




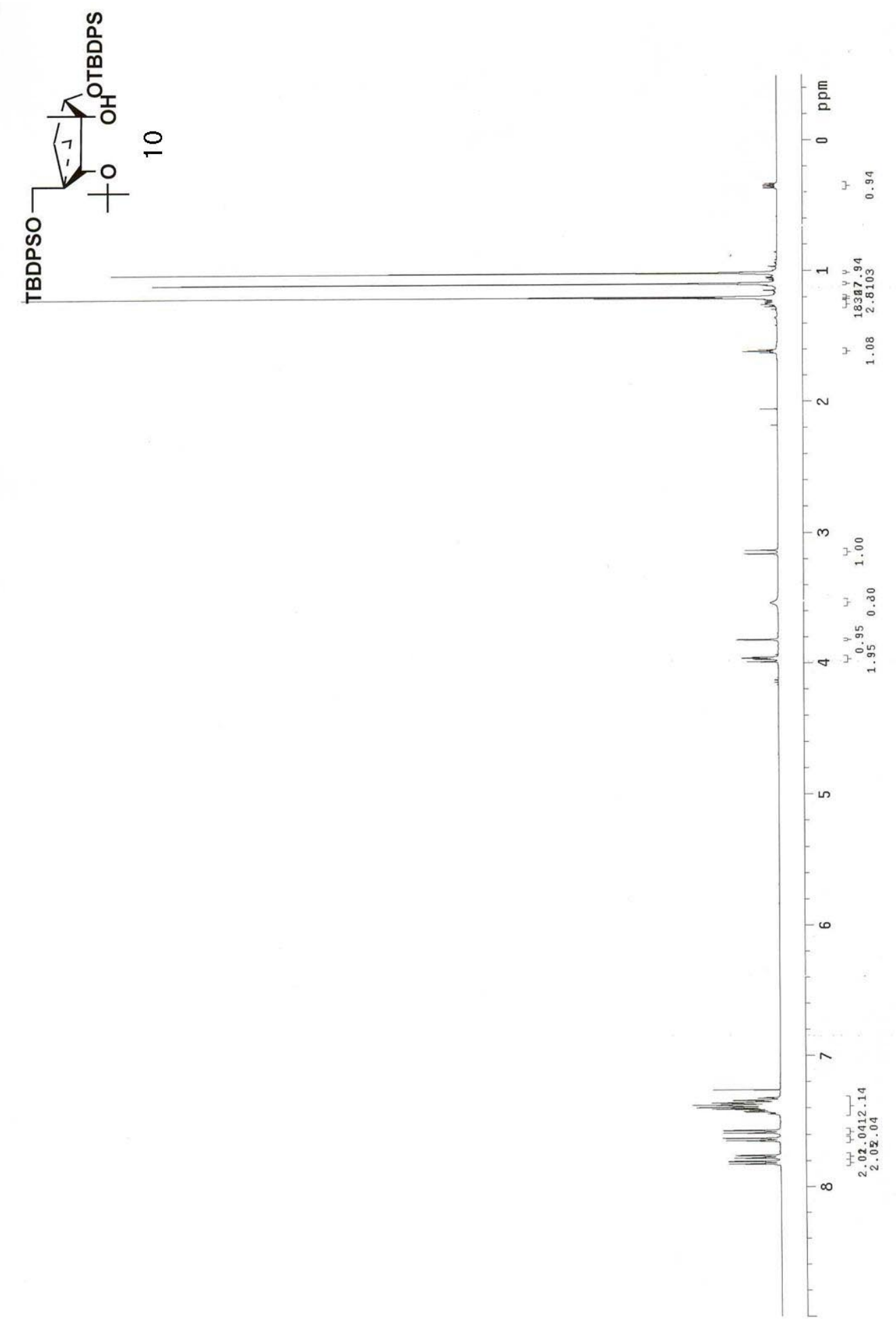




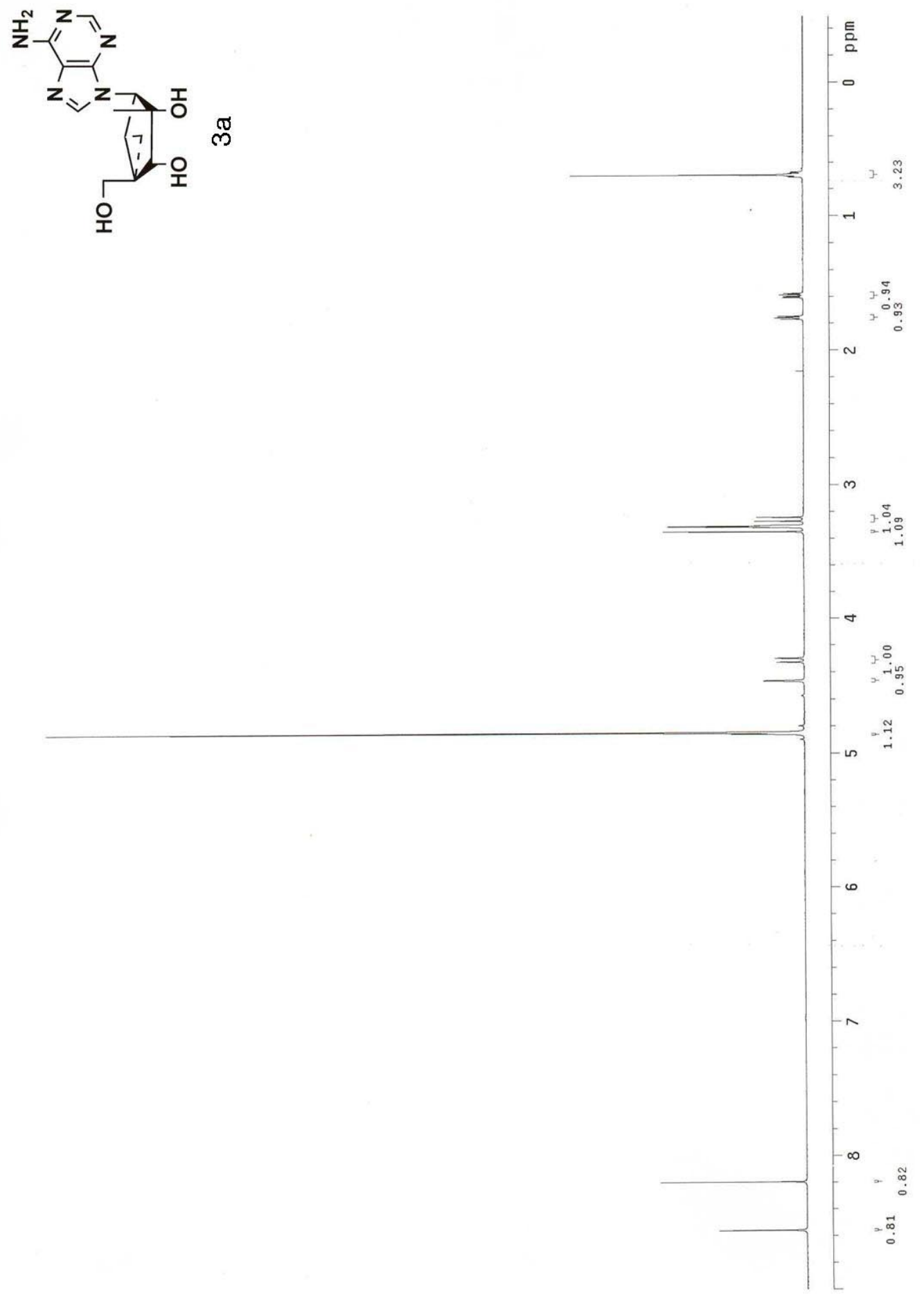




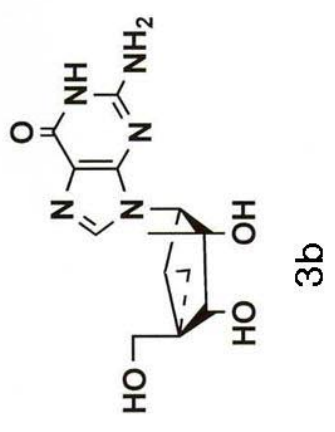

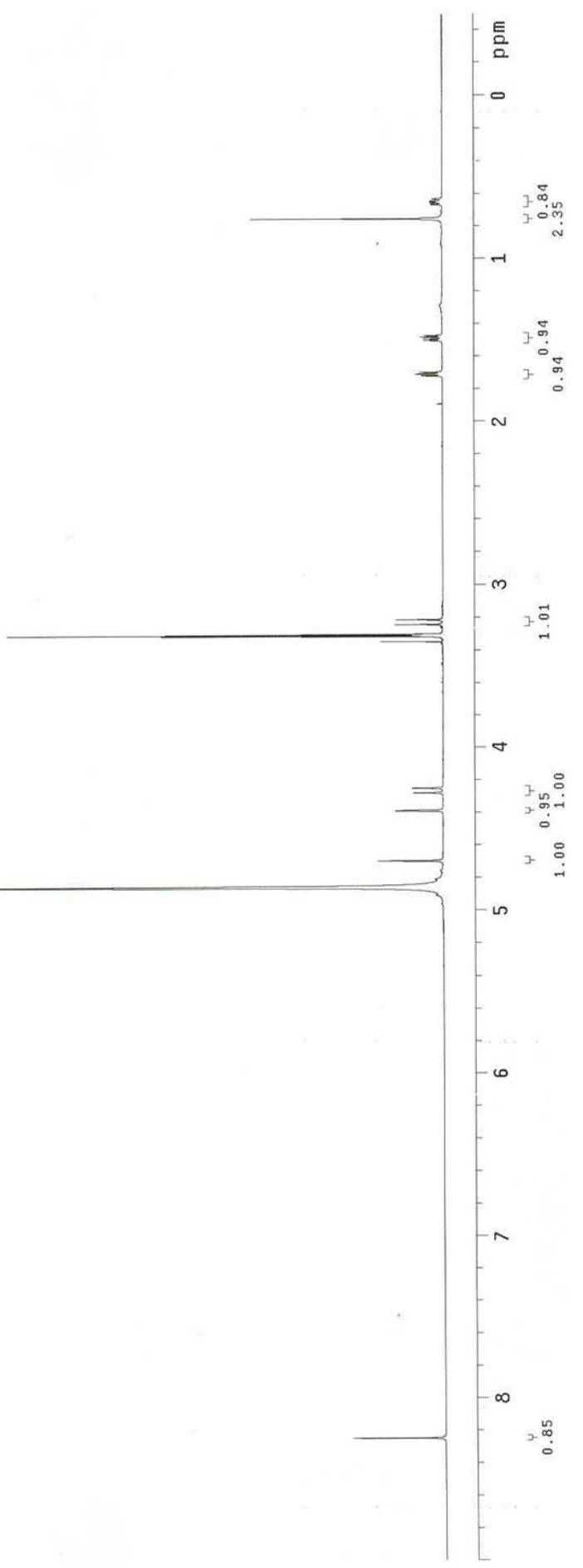



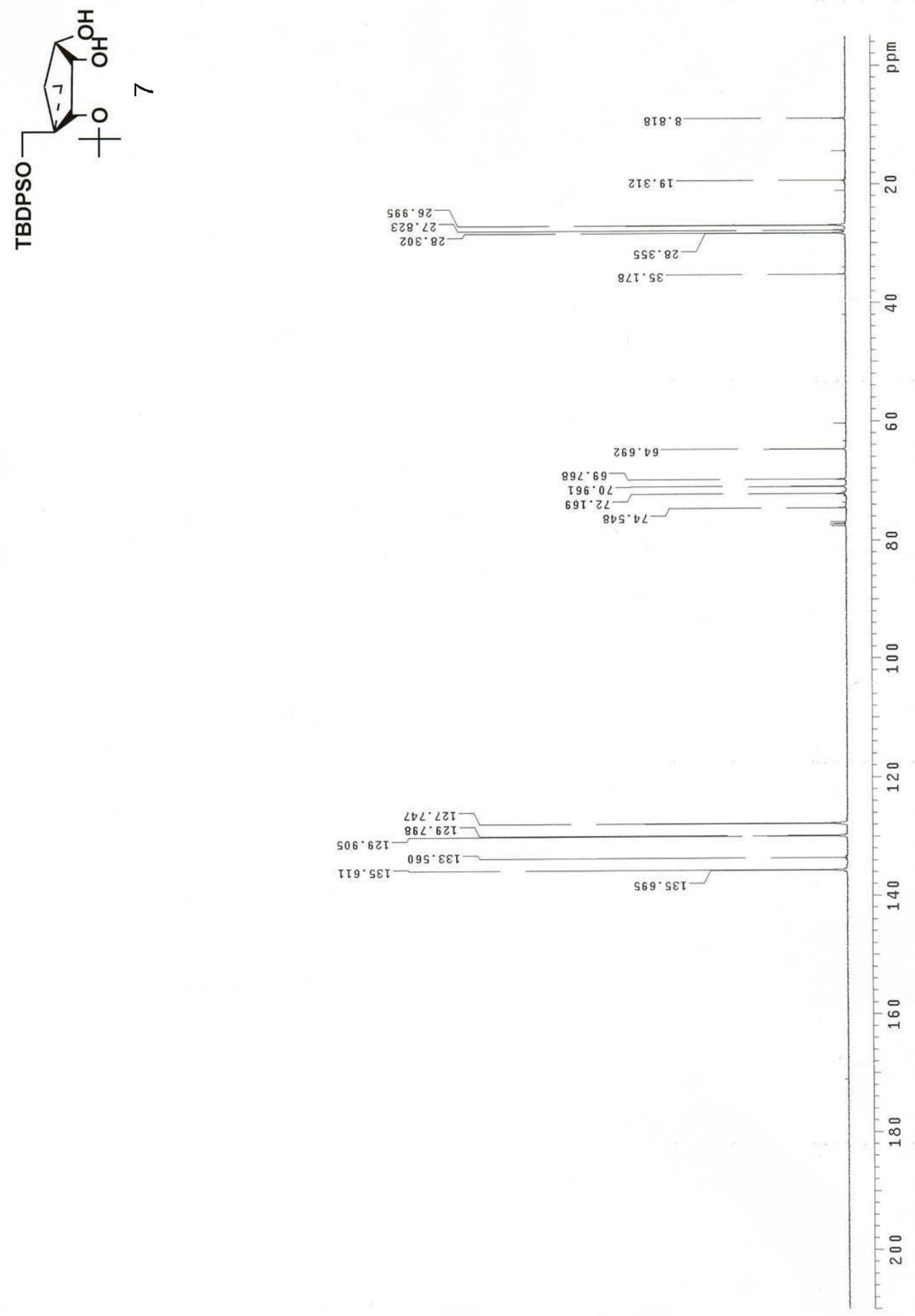

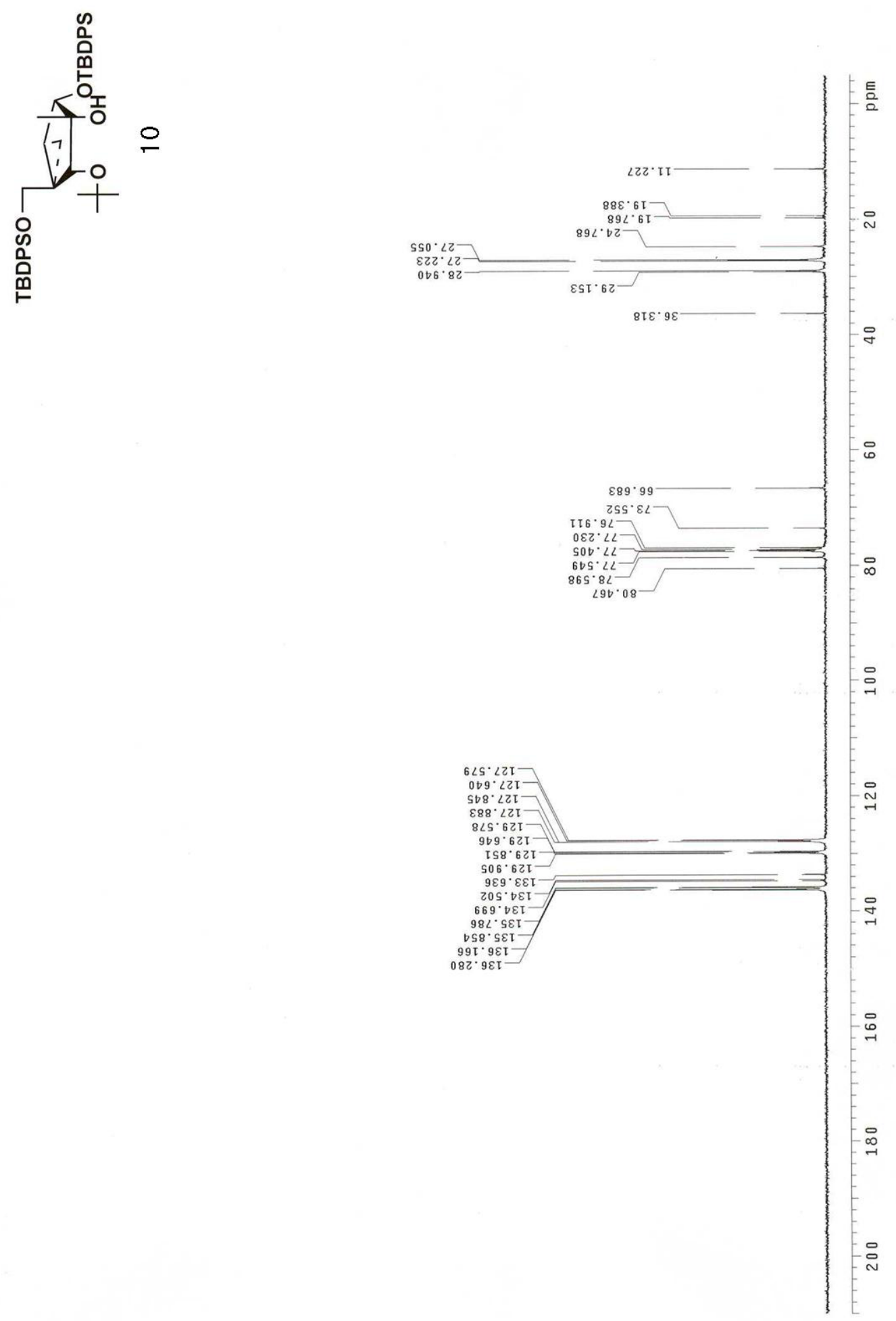

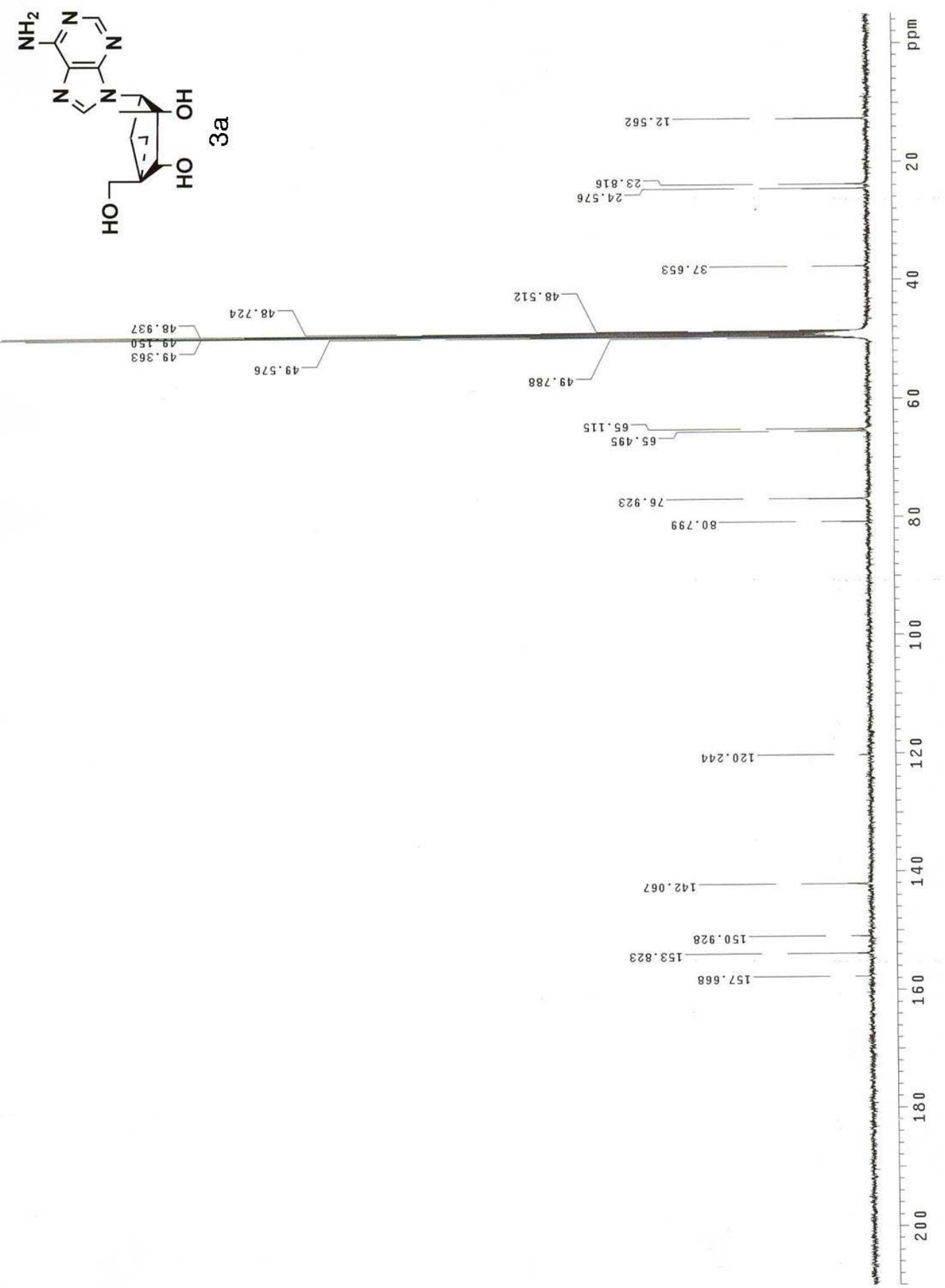

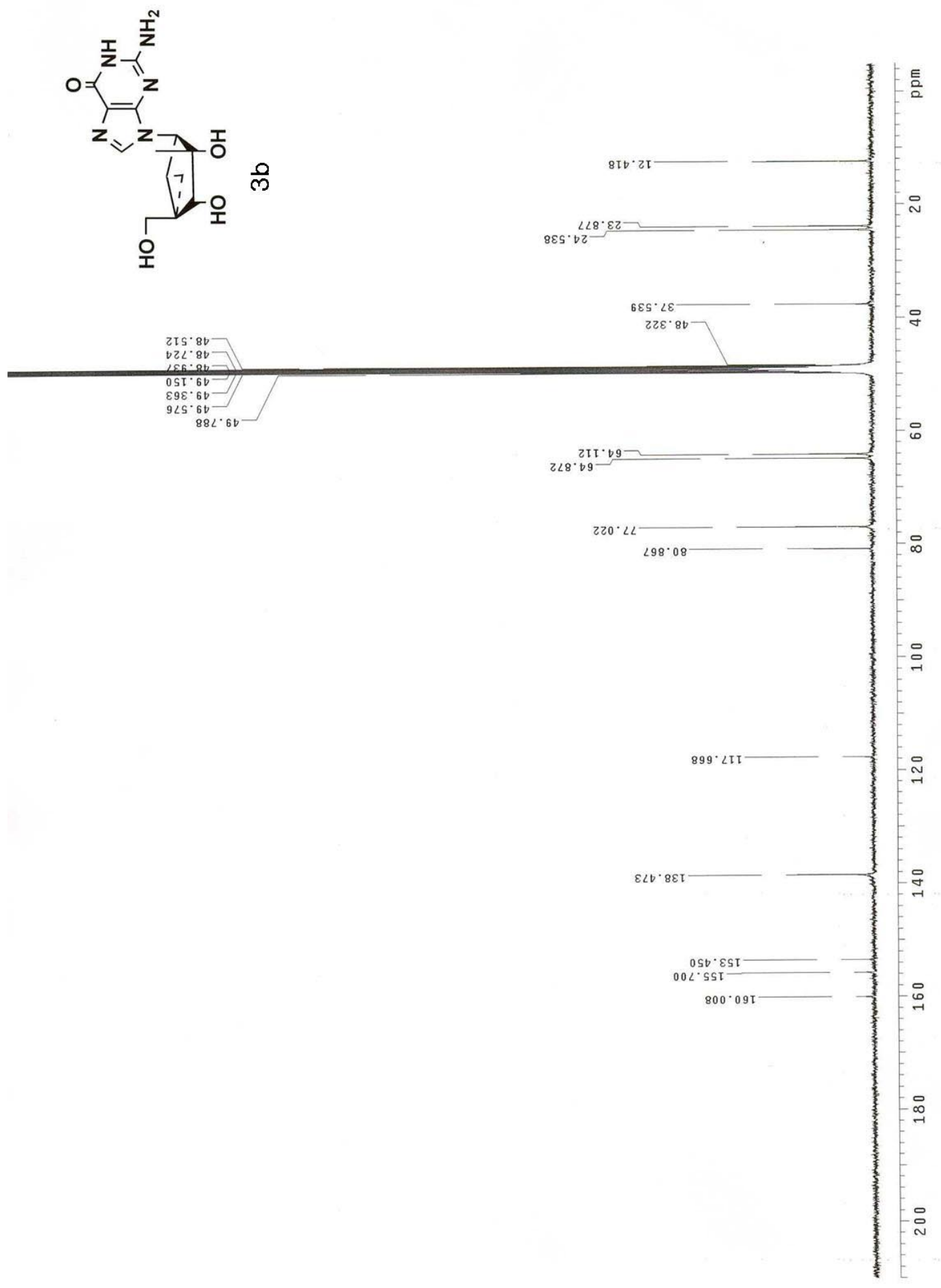